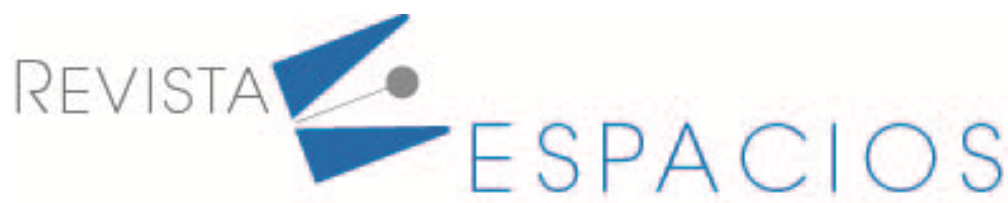

Vol. 42 (24) 2021 • Art. 3 (Especial Publicaciones académicas y COVID-19)

Recibido/Received: 10/12/2021 • Aprobado/Approved: 15/12/2021 • Publicado/Published: 30/12/2021

DOI: $10.48082 /$ espacios-a21v42n24p03

\title{
Información académica y pandemia
}

\author{
Academic information and pandemic
}

\author{
RANGEL-ALDAO, Rafael ${ }^{1}$
}

\begin{abstract}
Resumen
La pandemia transformó la industria editorial y la cooperación científica que produjo en meses, vacunas seguras y eficaces contra la COVID-19. ¿Para qué sirvió todo esto? La medición de la audiencia reveló una cobertura mundial; y Venezuela accedió a ciertas vacunas COVAX, pero no hay un plan nacional de vacunación; y el subregistro de datos sigue vigente. A escala global se lograron vacunas y antivirales, y estaremos mejor aún con una mayor cooperación que reduzca la desigualdad social del mundo.
\end{abstract}

Palabras clave: información, COVID-19, academia

\begin{abstract}
The pandemic transformed the publishing industry, and a scientific cooperation that in months produced safe and effective vaccines against COVID-19. What was all this for? The audience measurement revealed worldwide coverage; and Venezuela agreed to certain COVAX vaccines, but there is no national vaccination plan; and the data underreporting is still in effect. On a global scale, vaccines and antivirals were achieved, and we will be even better with greater cooperation that reduces social inequality in the world.

Key words: information, COVID-19, academy
\end{abstract}

\section{Introducción}

Entre tantas revelaciones de la COVID-19 destacan cómo surgieron inesperadas fuentes de acceso a la información científica y académica de la pandemia. Lo más novedoso, quizás, fue la transformación de la industria editorial científica con preimpresos o preprints en inglés, que facilitaron la publicación de artículos científicos en tiempo real, mientras se espera por la revisión definitiva de pares. De igual forma, tomaron liderazgo las bases de datos de información genética, como la secuenciación de genomas virales completos entre la plétora de variantes sucesivas del coronavirus o SARS-CoV-2. De allí que fuera posible en apenas semanas, a partir del 10 de enero 2020, establecer el diseño de las primeras candidatas a vacunas basadas en ARN mensajero, que luego se hicieron realidad a finales del año pasado como las de Pfizer/BioNTech y Moderna. El acceso libre a esas bases de datos es quizás el epítome de la colaboración científica mundial, sin precedentes en la historia.

En forma paralela, sin embargo, también surgió la desinformación en desmedro de medidas de contención y mitigación como las cuarentenas, el distanciamiento social y la protección personal, así como en la disposición a 
vacunarse por parte de millones de personas en el mundo entero. El costo ha sido inmenso tanto en políticas públicas fallidas, como en hospitalizaciones y muertes por más de cinco millones, con sucesivas ondas de morbimortalidad ante la pandemia, y de ello no escapan las naciones más desarrolladas y, por tanto, las mejor "preparadas" para combatir la pandemia. A dos años de Wuhan 2019, continúa la pandemia atemorizando la paz y prosperidad de mundo, ahora con la irrupción de la variante Omicron que, desde Botsuana se propagó a Sudáfrica, Europa, Asia, Oceanía y América. Lo peor es que la absoluta transparencia informativa de Sudáfrica con su palabra de alerta al mundo, más bien provocó el castigo por naciones avanzadas, mediante la prohibición de viajes hacia y desde ese país africano. La respuesta adecuada debió haber sido una mayor cooperación internacional y ayuda material a Sudáfrica, con vacunas, diagnósticos y secuenciación de ARN viral.

\section{Metodología: Análisis editorial de la literatura científica}

El mundo editorial científico experimentó ese torrente inédito de publicaciones a partir de enero 2020 (Cernile et al., 2021), producto de un alto grado de colaboración internacional jamás visto (Bernardo et al., 2021), con más de 200.000 artículos y preimpresos publicados en ese primer año 2020 (Else, 2020). De igual forma, la base de datos PubMed de la Biblioteca Nacional de Medicina de Estados Unidos, NLM, reportó 1.118 ensayos clínicos sobre COVID-19 en 2020-2021. La explosión informativa cobró vigor con los reportes en línea como los de ourworldindata un portal estadístico de libre acceso, desarrollado por la Universidad de Oxford, que "muestra el cambio de las condiciones de vida en todo el mundo, a través de gráficos y mapas interactivos." También destaca el portal de la Universidad Johns Hopkins de Estados Unidos, y su centro de coronavirus, con información cuantitativa en tiempo real sobre la pandemia en todas partes del mundo. Los diarios líderes como The New York Times ofrecen mapas interactivos sobre los casos de COVID-19 a escala global, así como información en línea sobre las campañas de vacunación con detalles sobre dosis y naturaleza de las vacunas.

Las redes sociales como Twitter se transformaron en fuentes importantes de información científica con investigadores vanguardistas como Eric Topol, un cardiólogo especialista en medicina digital e inteligencia artificial (Topol, 2019), que por ese canal se convirtió en uno de los principales divulgadores del avance contra la pandemia, con más de medio millón de seguidores a escala internacional. La red WhatsApp es otro medio importante de comunicación científica al punto que a través de sus chats varias organizaciones nacionales crearon lugares de intercambio de información sobre la pandemia, y el modo de combatirla a través de medidas como la contención y mitigación, distanciamiento social, fármaco vigilancia, secuenciación genómica del coronavirus y, sobretodo, de la naturaleza de las vacunas y la necesidad de un plan nacional de vacunación que en Venezuela nunca se ha hecho público si es que existe. Entre estas organizaciones que copan la audiencia científica del país destacan la Academia Nacional de Medicina, la Academia de Ciencias Físicas, Matemáticas y Naturales, Acfiman, la Sociedad Venezolana de Salud Pública, y Médicos por la Salud, entre otros.

Otras redes de información fiable como ResearchGate forman parte de lo que podría llamarse la "vitrina global" de la ciencia, con unos veinte millones de investigadores afiliados cuya obra completa aparece reseñada allí en tiempo real, al igual que GoogleScholar. Entre ambas redes es posible hacer un inventario de lo que publica cada científico de las ciencias biológicas en el mundo, así como el número de citaciones que recibe cada uno de los artículos de sus respectivos autores. La transparencia es total, lo cual debería ser un estímulo contra la charlatanería y la anti-ciencia de algunos medios y redes sociales que difunden información falsa o ambigua.

El libre acceso a estas redes científicas, así como a revistas en línea de nuevo cuño y alta calidad como las del grupo PLOS, Public Library of Science, es otra adición a la transparencia editorial. Este tipo de publicaciones y de otras como SciMago, Journal \& Country Rank, y otras bases de datos bibliográficas, como la internacional Scopus, y LILACS que cubre la literatura científica en ciencias de la salud en América Latina y el Caribe, contribuyen como fuentes de información bibliométrica a la llamada cienciometría, que se ocupa del cómputo de indicadores de 
desempeño como el factor de impacto para comparar revistas científicas según las citaciones de sus artículos año tras año: así como del llamado índice h que mide la productividad y el impacto de artículos tanto de revistas, autores e instituciones académicas (Hirsch, 2005).

\section{Resultados y discusión}

La opacidad oficial sobre la COVID-19 impuso en Venezuela importantes retos a la comunidad científica para difundir información veraz y accesible al gran público venezolano. A casi dos años del inicio de la pandemia y ante siete vacunas aprobadas por la Organización Mundial de la Salud, OMS, en Venezuela no se conoce a ciencia cierta el número de vacunados con las dos dosis de las vacunas existentes en Venezuela, tales como Sputnik-V, Sinopharm y Sinovac. De acuerdo con las estimaciones de Acfiman, para febrero 2021, las cifras oficiales de la pandemia representan apenas un $25 \%$ de los casos reales, e igual ocurre con el número de diagnósticos diarios mediante la prueba de PCR. El ocultamiento de la información contrasta con el de otros países latinoamericanos como Argentina, Brasil, Colombia, Chile, y México, por ejemplo, así como de otros países de la región cuyas autoridades dan reportes oficiales y veraces sobre el número de casos confirmados de coronavirus en la América Latina.

Un fenómeno similar de opacidad informativa ocurre con la efectividad y seguridad de las vacunaciones en Venezuela. Como muestra, apenas en julio 2021 se supo a través de una publicación científica de un grupo del Instituto Venezolano de Investigaciones Científicas, IVIC, que la vacuna rusa Sputnik-V confiere poca protección al ser administrada en una sola dosis (Claro et al., 2021). Nada de esta información aparece en los medios oficiales o siquiera en la prensa nacional.

Por el contrario a la información oficial, las academias y sociedades científicas venezolanas a partir de marzo 2020 hasta la presente fecha de fin de año 2021, tomaron la iniciativa de procesar y divulgar el curso de la pandemia en Venezuela mediante fuentes oficiosas como la Organización Mundial de la Salud, y grupos de derechos humanos, la Oficina Panamericana de Salud, OPS/OMS, las bases de datos internacionales ya citadas; así como artículos científicos y preimpresos de la plétora pandémica. El siguiente es un relato de cómo fue posible utilizar tecnologías de información y comunicación a través de distintos medios digitales, tanto académicos como periodísticos de alcance nacional e internacional.

La Academia Nacional de Medicina, ANM, por ejemplo, introdujo su portal digital a partir de mayo 2020, con dos secciones innovadoras que a partir de esa fecha ofrecen a diario información científica sobre la pandemia. En primer lugar, está la revista electrónica de libre acceso, CientMed, con varias categorías de información científica de frontera como los editoriales, tecnologías, y ciencias médicas. El lanzamiento de esta revista tuvo tanto éxito como para abarcar una audiencia mundial con 77.343 usuarios activos en 3.009 ciudades y 137 países de los cinco continentes, a través de 125.333 visitas en 93.961 sesiones. Las publicaciones de CientMed atrajeron el interés del 56,7\% de todas las consultas al Portal Digital, para un total de 71.009 vistas de esos 77.343 usuarios activos (Rangel-Aldao, 2021). CientMed, por ejemplo, significó un mecanismo de alerta a la ciudadanía sobre la falta de sustentación científica del DR10 y Carvativir, dos candidatos a medicamentos antivirales anunciados por el gobierno como estrategia contra la COVID-19. Los investigadores venezolanos tuvieron en CientMed una vía de publicación expedita, arbitrada, y de libre acceso a importantes trabajos sobre medicina tropical y tecnologías biomédicas de última generación. Las conferencias y publicaciones de CientMed se difundieron también a través de la red científica, ResearchGate, y significaron 3.261 lecturas a sus artículos y editoriales por parte de esta comunidad de alcance global. La revista contribuyó a la divulgación científica sobre los avances contra el COVID a través de 30 Sinopsis de CientMed durante el período agosto 2020 a marzo 2021. 


\section{Figura 1}

Entrada de la Sección COVID-19 del Portal

Digital de la Academia Nacional de Medicina

\section{El consorcio Harvard-Janssen-North Carolina, reporta su vacuna en Science: sí hay protección con anticuerpos neutralizantes contra Covid-19. 20/5/2020}

Deja un comentario / Covid-19, Vacunas / Por ANM

Compartir

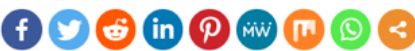

La pandemia mundial de COVID-19 causada por el virus SARS-CoV-2 convirtió el desarrollo de una vacuna en prioridad biomédica. En este estudio, desarrollamos una serie de candidatos a vacunas de ADN que expresan diferentes formas de la proteína espiga -Spike (S) SARS-CoV-2- y los evaluamos en 35 macacos rhesus. Los animales vacunados desarrollaron respuestas inmunes humorales y celulares, incluidos títulos de anticuerpos neutralizantes comparables a los encontrados en humanos convalecientes y macacos infectados con SARS-CoV-2. Después de la vacunación, todos los animales fueron desafiados con SARS-CoV-2, y la vacuna que codifica la proteína S de longitud completa produjo reducciones $>3.1 \mathrm{y}>3.7 \log 10$ en las cargas virales medias en el lavado broncoalveolar y la mucosa nasal, respectivamente, en comparación con la simulación, control S. Los títulos de anticuerpos neutralizantes provocados por la vacuna se correlacionan con la eficacia protectora, lo que sugiere un correlato inmune de protección. Estos datos demuestran la protección de la vacuna contra el SARS-CoV-2 en primates no humanos

\section{PDF}

A

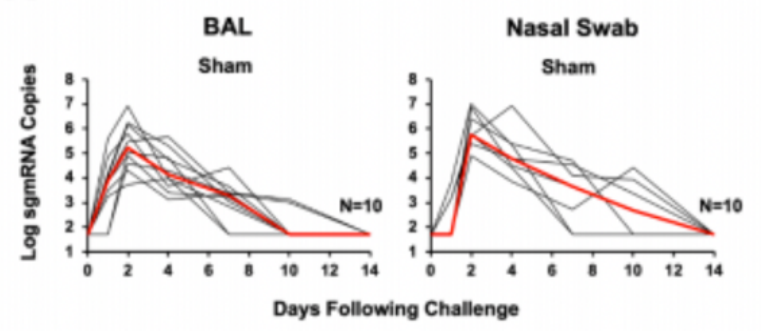

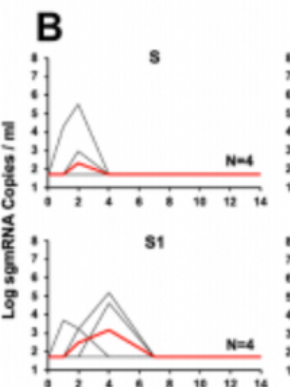
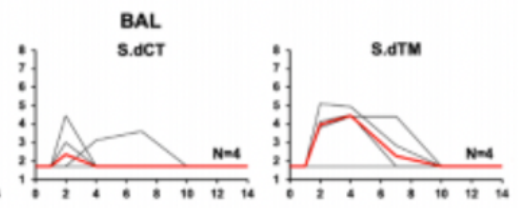

RBO
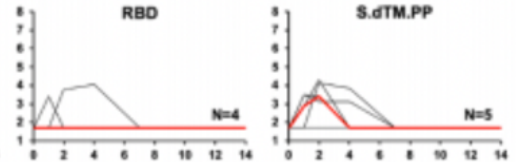

Fuente: https://academianacionaldemedicina.org/publicaciones/covid-19/cv-el-consorcio-harvard-jenssen-north-carolinareporta-su-vacuna-en-science-si-hay-proteccion-con-anticuerpos-neutralizantes-contra-covid-19/

La sección COVID-19 del Portal Digital de la ANM, fue la base de las Sinopsis semanales presentadas durante el período agosto 2020 a marzo 2021, mediante un proceso de síntesis de centenares de artículos y preimpresos sobre la pandemia registrados en la base de datos bibliográficos de PubMed. COVID-19 fue dividida en las siguientes cuatro categorías, Protección y Mitigación, Diagnóstico, Tratamiento, y Vacunas, para luego, a partir de abril 2021, agregar en forma sucesiva, Síndrome post-COVID-19 y Aspectos Varios. Cada entrada de esas categorías constaba de una combinación de síntesis conceptual en español, con ilustraciones originales extraídas de los artículos científicos revisados, acompañados del PDF del trabajo original, como se muestra en la Figura 1.

La Figura 2 ilustra la composición de la primera Sinopsis semanal publicada en el Portal Digital la semana del 3 al 11 de agosto 2020, en comparación con la Sinopsis 30 del 23/02 al 01/03/2021, es decir, en el lapso de seis meses de la pandemia. 


\section{Figura 2}

Comparación del contenido de la Sinopsis 1 de agosto 2020, con la portada de la Sinopsis 30 de marzo 2021

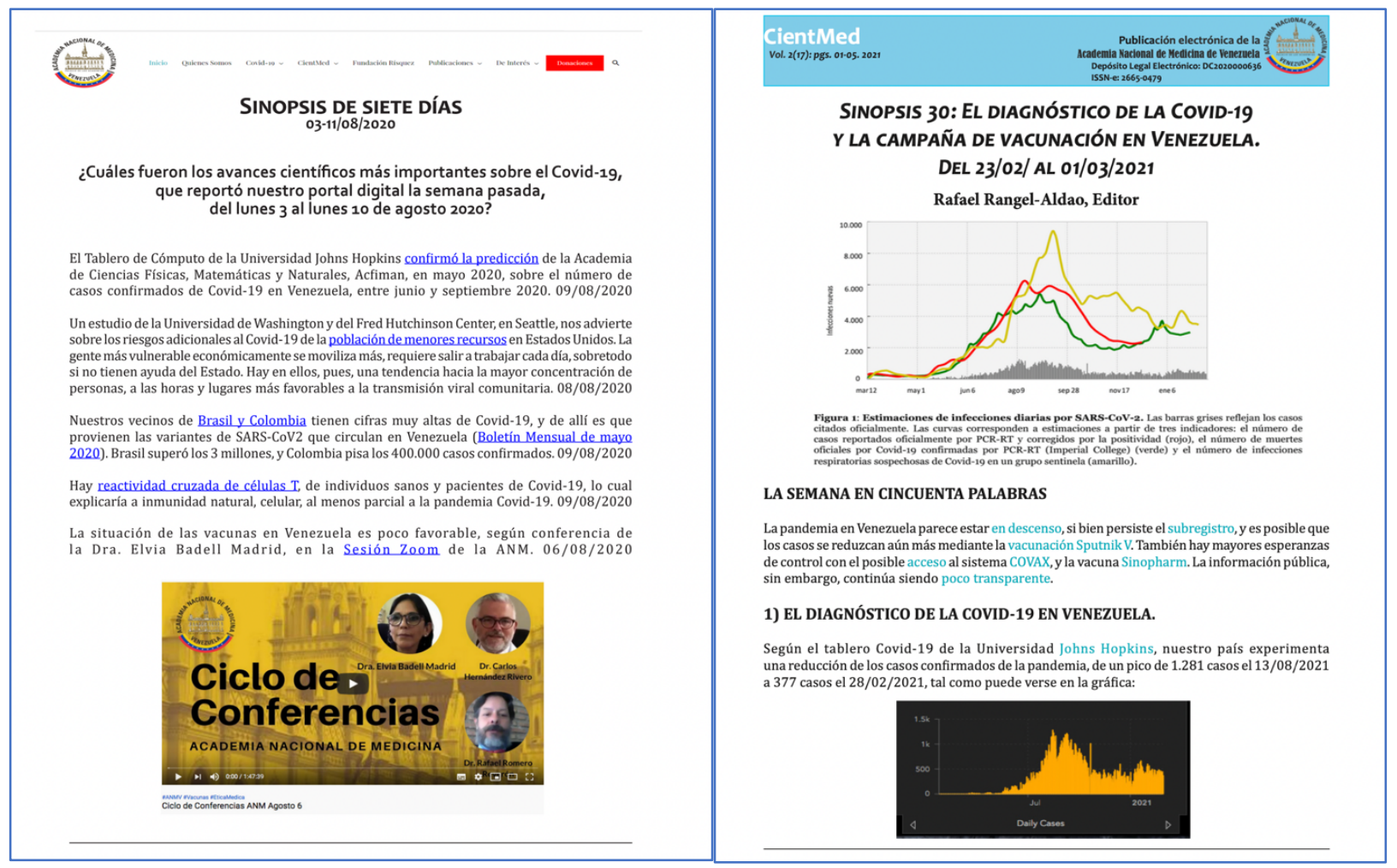

Fuente: http://academianacionaldemedicina.org/

La Figura 2 también permite visualizar el cambio del énfasis en las investigaciones sobre la pandemia, del impacto inicial en países como Estados Unidos y vecinos latinoamericanos como Brasil y Colombia, hacia cómo se desarrollaba la campaña de vacunación contra COVID-19 en Venezuela. En ambas gráficas también es posible distinguir la variedad de medios usados para difundir la información científica mediante una combinación de conferencias y videos en la Sinopsis 1, con la síntesis conceptual y visual de la Sinopsis 30. Cada Sinopsis constaba también de un PDF con la información e hipertextos que conectaban a los trabajos originales como fue referido arriba.

El cambio de énfasis de las investigaciones sobre COVID-19 entre agosto 2020 y marzo 2021, según el contenido de las Sinopsis semanales de CientMed, fue todavía más evidente al aplicar el algoritmo de nube de palabras que permite el análisis semántico computacional a través del cómputo de las palabras más frecuentes en un texto (Binucci et al., 2016). La Figura 3 ilustra el contraste entre ambas semanas durante el período de seis meses entre agosto 2020 y marzo 2021.

En la Sinopsis 1 de marzo 2021 destaca, además del término COVID-19, la palabra "confirmados" que se refiere a los casos de la pandemia en Venezuela, Colombia y Brasil. Sobresalen también las palabras Academia, Acfiman, portal, matemáticas, científicos riesgos, y boletín. En la Sinopsis 30, de marzo 2021 es evidente el predominio, después de la COVID-19, las palabras vacunación, vacunas, Venezuela, nacional, CientMed, Sinopsis, y en menor grado, "casos" y "pruebas". 


\section{Figura 3}

Comparación semántica de la Sinopsis 1 de agosto 2020, a la izquierda; con la Sinopsis 30 de marzo 2021, a la derecha de la gráfica.
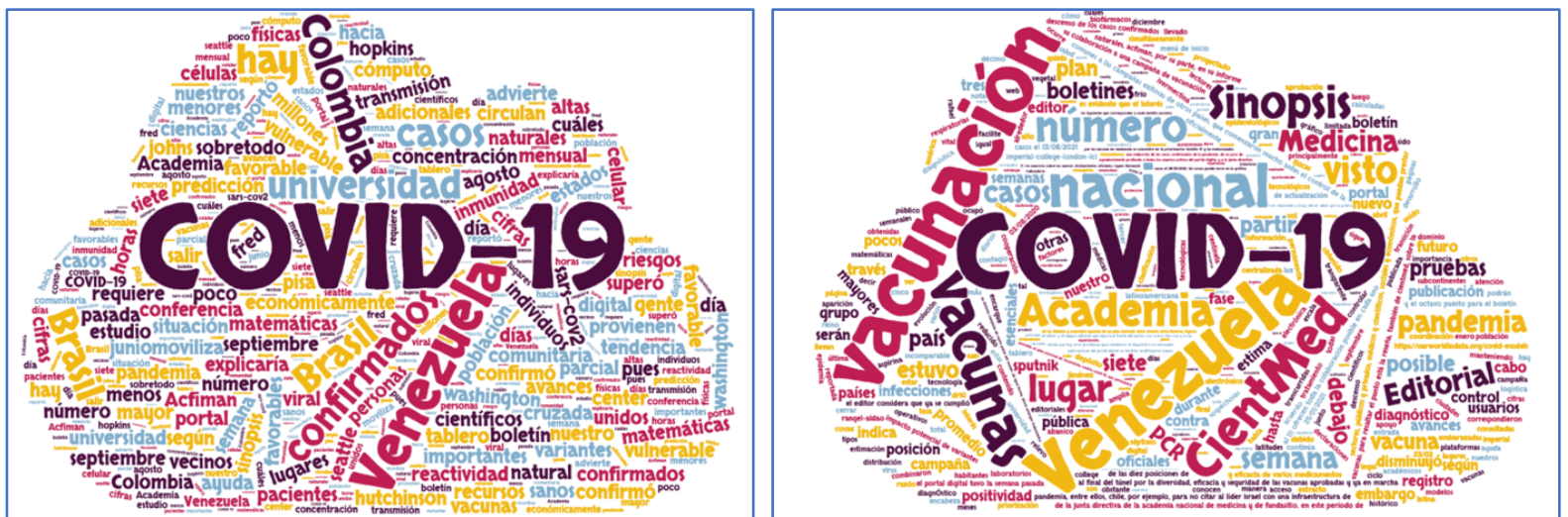

Fuente: https://www.nubedepalabras.es/

La palabra "boletín" que apenas aparece en la Sinopsis 1, producto de un comunicado de Acfiman, incrementa su frecuencia como "boletines", en la Sinopsis 30, que se refiere a otra publicación importante de la ANM entre enero 2021 y el 12 de diciembre 2021, en la forma de 45 boletines. Este tipo de publicación tuvo como objetivo alertar a la población y al gobierno sobre la necesidad de un plan nacional de vacunación, así como de llenar los requisitos de rigor y las precauciones que requieren las vacunas contra la COVID-19. Al respecto, la Figura 4 ilustra el contenido semántico de cuatro boletines clave espaciados entre febrero y diciembre 2021. Del análisis de este gráfico es obvio que la ANM asumió la difícil tarea de difundir información importante y veraz para advertir al país sobre un amplio espectro de las medidas para neutralizar la pandemia a través de una combinación de medidas de salud pública como el diagnóstico y la vacunación masiva, en especial para los más vulnerables, como embarazadas, niños, y adultos mayores. También destacan las advertencias sobre el riesgo de usar candidatos a vacunas no registradas como seguras y eficaces, como es el caso de las cubanas Abdala y Soberana 2.

El siguiente medio de comunicación de la ANM ha sido la revista emblemática desde la fundación de esta corporación en 1904, la Gaceta Médica de Caracas, GMC. En este sentido y durante 2020-2021, la GMC publicó su primer artículo sobre COVID-19 en el número de julio-septiembre 2020, mediante una revisión bibliográfica de F.J. Tapia y O.L. Rodríguez sobre la patogenia de la enfermedad (Tapia, 2020).

En noviembre 2020, GMC dedica un suplemento especial a la pandemia editado por M. Guzmán, quien en un editorial resume el contenido de ese suplemento en los siguientes términos:

"Este año 2020 ha sido marcado por una pandemia de caracteres históricos, la infección por SARSCoV-2. Están incluidos en este número varios artículos originales que describen experiencias venezolanas: David Forero y su grupo quienes describen la experiencia en el Hospital Universitario de Caracas; el trabajo de Andrea Mujica y colaboradores describiendo el Síndrome de Guillain Barré como complicación; una extensa revisión sobre el problema de la coagulopatía por los Drs. Müller y Soyano, que incluye alguna experiencia de la Clínica El Ávila de Caracas. Rísquez y Fernández abordan el tema de la situación de salud en el país en el momento de la llegada del COVID-19, y dejan en evidencia la difícil situación. Dos trabajos adicionales y muy importantes sobre el tema vienen del exterior, e incluyen la observación de De Sanctis y su grupo sobre el papel regulador de Ios microRNA y el trabajo de Aceituno y asociados sobre el manejo del dolor crónico en pacientes con COVID-19." 
Figura 4

Análisis semántico computacional mediante nubes de palabras, de cuatro boletines de la Academia Nacional de Medicina de Venezuela. Panel superior izquierdo, contenido semántico del Boletín 7 del 10 de febrero 2021, sobre las medidas de precaución en la vacunación de embarazadas. A la derecha está el resultado del

Boletín 20 del 23 de marzo 2021 sobre la prevención ante candidatos experimentales a vacunas cubanas como Abdala y Soberana, aún no autorizadas como eficaces y seguras. Panel inferior izquierdo, Boletín 36 del 5 de julio 2021 sobre la vacunación en niños. Al margen derecho está el Boletín 44 del 6 de diciembre sobre la variante Omicron.
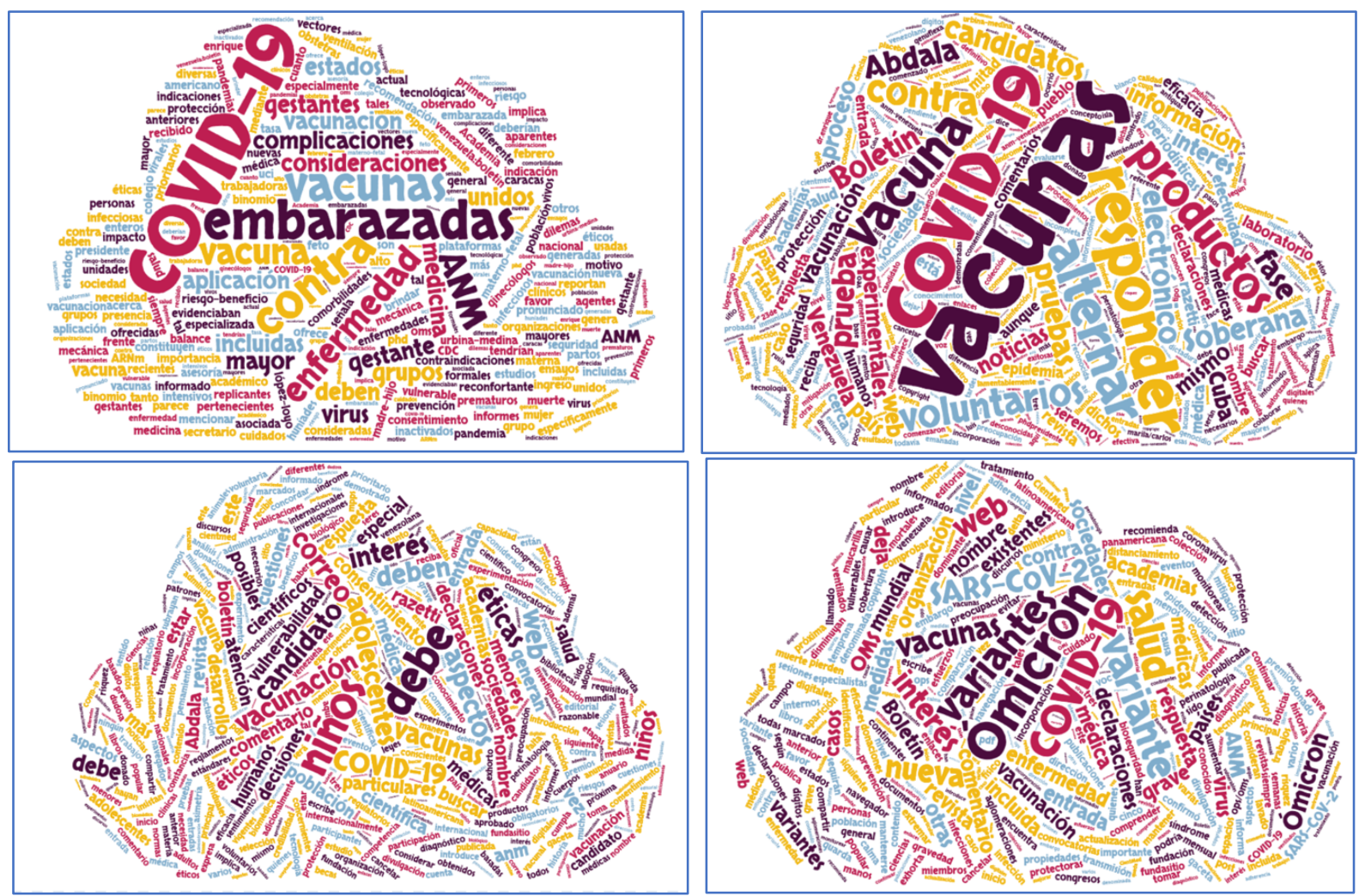

Fuente: https://www.nubedepalabras.es/

En diciembre 2020 la GMC publica otro suplemento, el №. 2, dedicado a la COVID-19 en América Latina, esta vez en inglés, editado por M.J. González y M. Fernández-Silano, quienes describen el objetivo de ese número especial de la siguiente manera:

"Tres propósitos guiaron el desarrollo de este suplemento. El primero fue la importancia de documentar los diversos impactos que ha tenido la pandemia en la región. A pesar de la velocidad de los acontecimientos que han conducido al surgimiento de múltiples áreas de conocimiento nuevas, a menudo cambiantes, resulta imperativo ordenar y analizar estos cambios, con alta probabilidad de que se modifiquen en breve. El segundo propósito, fue examinar dentro de las limitaciones de información y recursos impuestos por la pandemia, las lecciones aprendidas, especialmente porque, si se comunican, pueden ser útiles en otros contextos, dentro y fuera de la región. Finalmente, el tercer propósito fue identificar los desafíos y nuevas perspectivas requeridas para las próximas etapas del control de la pandemia, especialmente en lo que puede significar para la investigación y prioridades de intercambio de los centros académicos."

Los siguientes artículos de la GMC sobre COVID-19 aparecieron en el número de abril-junio 2021, en particular sobre el efecto de la pandemia en embarazadas. El siguiente artículo sobre COVID-19 fue publicado en el número de GMC de julio-septiembre 2021, de un conjunto de coautores cuyo líder fue L. Sánchez Traslaviña, cuyo 
objetivo fue, "Evaluar las características clínicas generales, nivel de atención requerida, estancia hospitalaria y mortalidad en pacientes hospitalizados con COVID-19 en un año de pandemia". Se trató de un estudio observacional a base de historias clínicas de un hospital privado de Caracas, cuyos resultados de medio millar de pacientes reveló que, "La estancia hospitalaria se mantuvo estable en el año, mientras que la mortalidad disminuyó progresivamente entre agosto 2020, con un 26,4 \% y la menor en marzo con un 5,4 \% (SanchezTraslaviña, 2021). En el número de GMC, Suplemento No. 3 de septiembre 2021, la revista publicó otro artículo relacionado con la lactancia y COVID-19 cuya autora fue D. Rosillón, con la siguiente conclusión (Rosillón, 2021),

"Hasta el momento, en cuanto a las vacunas empleadas en América Latina, incluyendo Venezuela, dentro de ellas la vacuna Sputnik-V, los fabricantes se muestran a favor de no suspender la lactancia, a pesar de no tener suficientes estudios en mujeres lactantes, lo que corresponde a la vacuna COVID19 BIBP (Sinopharm) estas vacunas contienen virus muertos, sin capacidad de infección. La OMS no recomienda suspender la lactancia materna después de la vacunación."

El Portal Digital de la ANM desde su inicio en mayo 2020, se conectó en línea a ciertos algoritmos analíticos de Internet que usan inteligencia artificial como es el caso de Google Analytics,

“...una herramienta de Google lanzada el 14 de noviembre de 2005, que ofrece información agrupada del tráfico que llega a los sitios web según la audiencia, la adquisición, el comportamiento y las conversiones que se llevan a cabo en el sitio web."

Figura 5

Dinámica de la audiencia mensual del Portal Digital de la ANM en el primer mes de su lanzamiento entre abril y mayo 2020

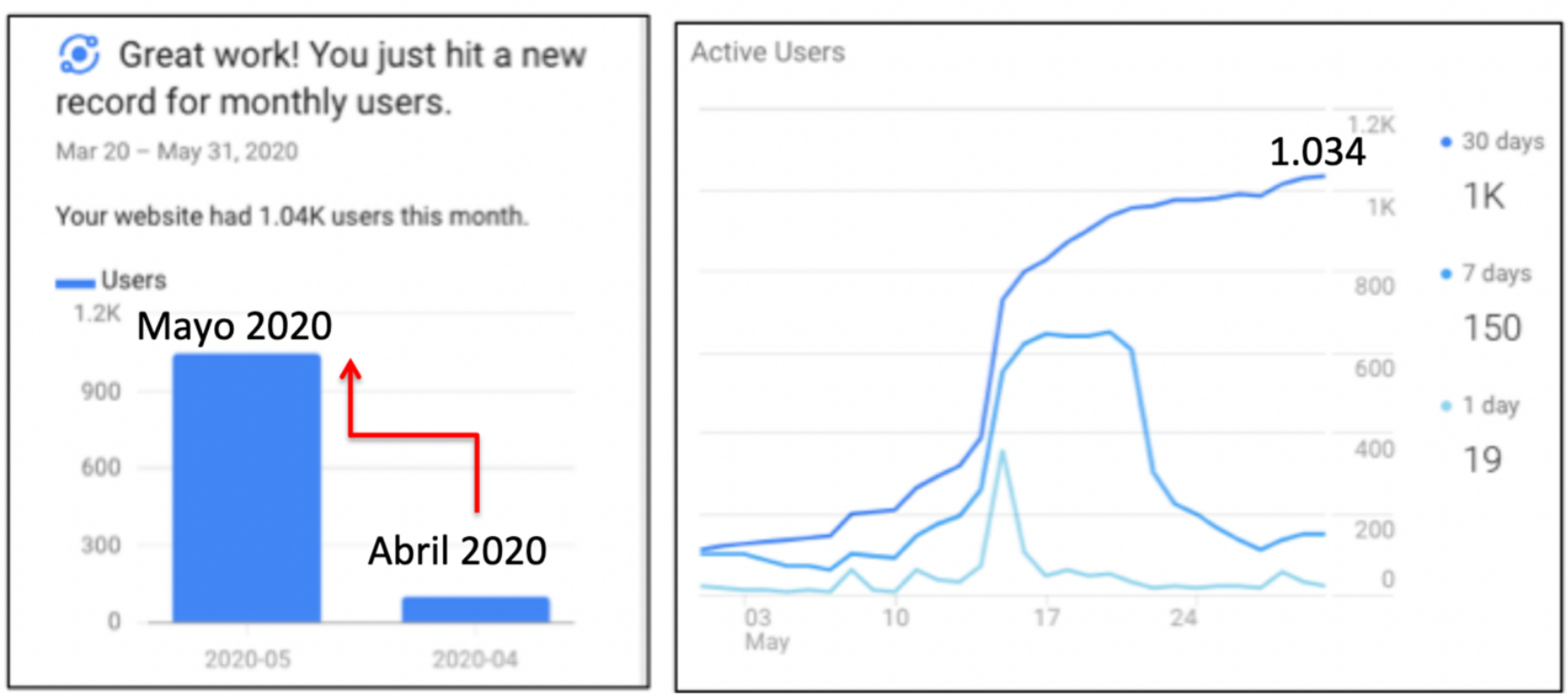

Fuente: (Rangel-Aldao, 2021)

De esta manera, se pudo determinar cómo de una audiencia presencial de unos treinta académicos que asistían cada semana a las sesiones de la ANM en el Palacio de las Academias, hasta marzo 2020, el Portal Digital hizo que aumentara la audiencia en forma exponencial hasta llegar en 2021 a las cifras referidas en la sección precedente. Es decir, a casi 80.000 usuarios de más de un centenar de países de los cinco continentes, de tal suerte que a través de Google Analytics fue posible establecer una métrica del alcance científico de la ANM en cuanto al destino de sus publicaciones en el mundo, a través de este canal electrónico y masivo de comunicación. (Rangel-Aldao, 2021) 
La Figura 5 ilustra en detalle el despegue de la audiencia del Portal Digital desde su inicio (Rangel-Aldao, 2021), y cómo fluctuó a diario la audiencia del Portal Digital, una tendencia a escala internacional que se mantuvo a través de todo el primer año de la pandemia hasta inclusive al primer trimestre de 2021, como lo indica la Figura 6, extraída de una presentación del autor en el XIX Congreso Venezolano de Ciencias Médicas, el 18 de septiembre 2021.

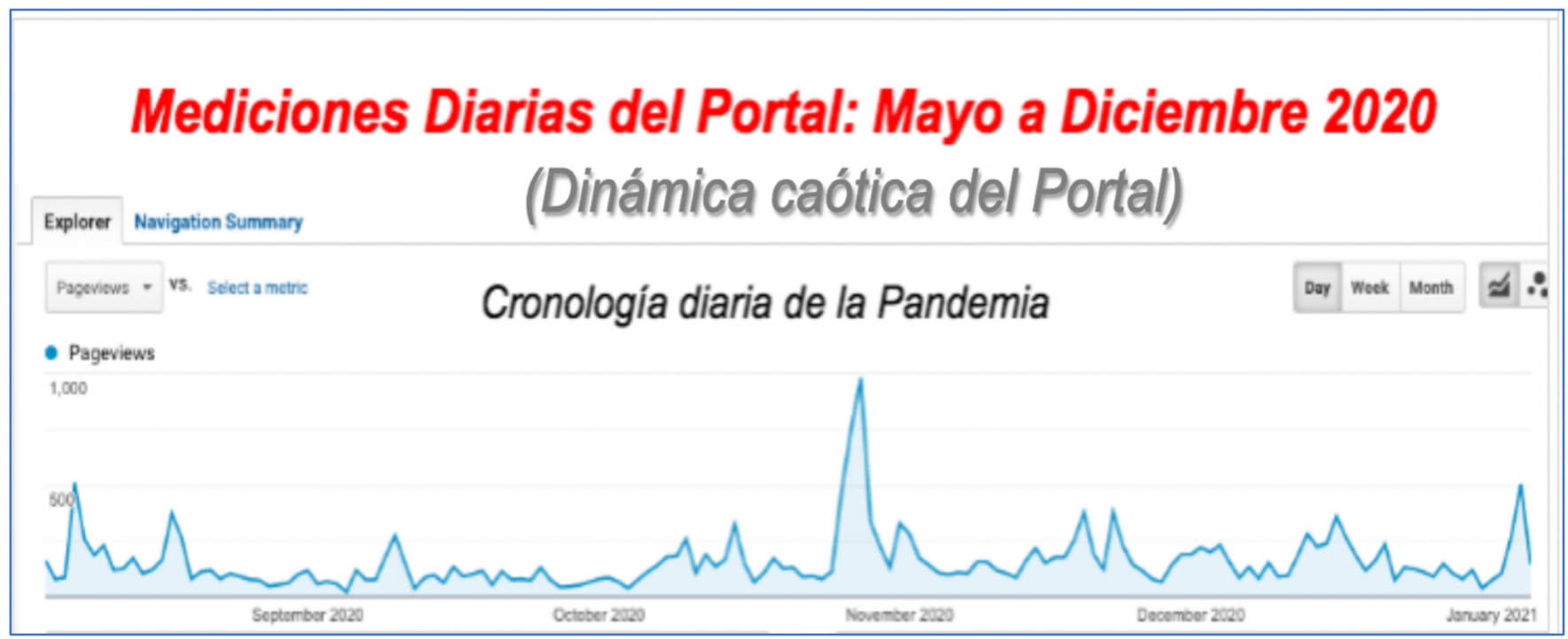

Fuente: Google Analytics

La dinámica caótica de la audiencia del Portal Digital, con picos y valles casi a diario, es típica de los sistemas complejos de información biológica, lo que asegura una mayor flexibilidad de respuesta ante los cambios del entorno (Goldberger et al., 2002). En el caso del Portal Digital de la ANM, el análisis posterior de la data, correspondiente a picos y valles mostrados arriba reveló la naturaleza de lo que llamó la atención del usuario. Por ejemplo, el pico principal que aparece en la Figura 6 se debió a un editorial de quien escribe, del 26 de octubre 2020, sobre el anuncio oficial de un supuesto medicamento contra la COVID-19, el ya referido y denominado DR10. Ese editorial resaltó la noticia en los siguientes términos:

"La noticia oficial no ofrece datos sobre la naturaleza química o farmacológica de la molécula DR10, apenas se limita a señalar que es eficaz contra el coronavirus, SARS-CoV-2_... No hay detalle alguno sobre los ensayos preclínicos y clínicos de seguridad y eficacia, de fase 0 a 4, ni tampoco si existen publicaciones respecto al compuesto DR10. Con el propósito de orientar a la comunidad médica del país, y en general a todos los usuarios del Portal Digital, a continuación, se explican ciertos detalles técnicos de la escueta noticia oficial sobre el tratamiento anti Covid-19, referido por el gobierno nacional."

Una noticia similar sobre otro anuncio oficial, pero del 25 de enero 2021, ocupó el primer lugar de lo más visto en el Portal Digital hasta la fecha de entrega de este ensayo, el 15 de diciembre 2021. Se trata del editorial de este mismo autor, en CientMed, con el título de "Nota preliminar sobre el antiviral Carvativir como medicamento para el COVID-19." La nota informa al público de la siguiente manera:

"Hay una sola conclusión válida con la información que hasta ahora es del dominio público: los extractos de tomillo [como el Carvativir], incluyendo muchos de los aceites esenciales derivados de 
esa planta, tienen el potencial terapéutico contra coronavirus, sin embargo, es prudente esperar por mayores datos de las pruebas del Carvativir, según los protocolos internacionales descritos arriba para calificar como candidato a medicamento anti Covid-19. Esperemos que así sea, y se convierta en realidad esta futura noticia."

La nota sobre el Carvativir fue también noticia de primera plana de la edición electrónica del 28 de marzo 2021, del diario español, El País, el de mayor audiencia global y uno de los diez más prestigiosos de mundo.

\section{Conclusiones}

A dos años del inicio de la pandemia en Wuhan (Liu et al., 2020), la pregunta obligada es ¿para qué sirvió esta plétora de información científica en Venezuela, y el mundo (Mehta \& Shukla, 2022); más aún con la aparición de variantes del coronavirus que resisten a las vacunas (Hacisuleyman et al., 2021)? La respuesta es compleja ante una causalidad múltiple, en particular cuando la pandemia sigue y seguirá vigente en 2022, y no solo por variantes como la Omicron (Petersen et al., 2021), sino por factores sociales como la inequidad de acceso a la vacunación masiva en los países menos favorecidos, y la desinformación por redes y medios de comunicación que alimenta la resistencia humana a vacunarse (Benoit \& Mauldin, 2021). No obstante, hay al menos dos vías de abordaje para esclarecer el impacto de la información científica, especialmente en Venezuela. La primera vía es tecnológica a través de la medición de la audiencia que recibe la información científica de fuentes como, por ejemplo, de la ANM, cuyo portal digital, como está explicado arriba, dispone de una métrica adecuada para tales fines. La segunda ruta de abordaje al impacto de la información sobre la pandemia, es mediante la verificación factual del efecto de las políticas públicas y medidas de control y prevención sanitaria tanto a escala nacional como global. En este caso, la data es relativamente transparente, de fácil acceso y gran fiabilidad a través de la internet y ciertas redes sociales.

Es evidente a través del análisis de las noticias que lideraron el interés de la audiencia del Portal Digital en 20202021, así como de otras similares relativas a la COVID-19, que el público demanda información científica con detalles técnicos, pero en un lenguaje corriente y entendible, para recibir la debida orientación hacia la prevención y control de la pandemia.

El análisis fáctico de lo alcanzado por las noticias científicas en nuestro país, sin embargo, no es el más alentador (Loyo et al., 2021). Si bien es evidente que el gobierno central logró el acceso a las vacunas COVAX, como las chinas Sinopharm y Sinovac, del grupo de las siete aprobadas por la OMS, aún no hay información fehaciente sobre la campaña de vacunación contra la COVID-19, pues no existe un plan nacional que sea del dominio público a pesar de los 45 boletines de orientación de la ANM, referidos arriba. Según la base de datos Ourworldindata a dos años de la pandemia apenas un $34 \%$ de la población venezolana recibió las dos dosis de las vacunas, que incluye además de las chinas, a la Sputnik-V.

En general, en Venezuela continua el subregistro tanto de los casos confirmados como del número de vacunados con las dos dosis, y continúan vigentes las deficiencias aparentes en el seguimiento de las variantes del coronavirus, mediante la secuenciación genómica de aislados virales. Este punto es muy importante y demanda la participación de todas las instituciones científicas del país con capacidad de análisis genómico, y no como hasta ahora que ha sido una labor titánica y heroica de un laboratorio del Instituto Venezolano de Investigaciones Científicas, junto al del Instituto Nacional de Higiene, "Rafael Rangel" (Jaspe et al., 2021; Loureiro et al., 2021; Ortega et al., 2021).

En el ámbito global, el impacto de la información científica es más que evidente, y no solo por el revolucionario desarrollo de las distintas vacunas y antivirales contra la COVID-19 (Asselah et al., 2021; Woldemeskel et al., 2021), sino también por los novedosos métodos de diagnóstico viral (Taleghani \& Taghipour, 2021), además las 
medidas asertivas de control y mitigación de muchos gobiernos que lograron reducir la carga de la pandemia. El panorama futuro, sin embargo, dista mucho de ser positivo mientras no se potencie y acelere la cooperación mundial para reducir la desigualdad social del acceso a la vacunación y diagnóstico masivo en los países más pobres.

\section{Referencias bibliográficas}

Asselah, T., Durantel, D., Pasmant, E., Lau, G., \& Schinazi, R. F. (2021). COVID-19: Discovery, diagnostics and drug development. J Hepatol, 74(1), 168-184. https://doi.org/10.1016/j.jhep.2020.09.031

Benoit, S. L., \& Mauldin, R. F. (2021). The "anti-vax" movement: a quantitative report on vaccine beliefs and knowledge across social media. BMC Public Health, 21(1), 2106. https://doi.org/10.1186/s12889-021$12114-8$

Bernardo, T., Sobkowich, K. E., Forrest, R. O., Stewart, L. S., D'Agostino, M., Perez Gutierrez, E., \& Gillis, D. (2021). Collaborating in the Time of COVID-19: The Scope and Scale of Innovative Responses to a Global Pandemic. JMIR Public Health Surveill, 7(2), e25935. https://doi.org/10.2196/25935

Binucci, C., Didimo, W., \& Spataro, E. (2016). Fully dynamic semantic word clouds 2016 7th International Conference on Information, Intelligence, Systems \& Applications (IISA),

Cernile, G., Heritage, T., Sebire, N. J., Gordon, B., Schwering, T., Kazemlou, S., \& Borecki, Y. (2021). Network graph representation of COVID-19 scientific publications to aid knowledge discovery. BMJ Health Care Informat, 28(1), e100254. https://doi.org/10.1136/bmjhci-2020-100254

Claro, F., Silva, D., Rodriguez, M., Rangel, H. R., \& de Waard, J. H. (2021). Immunoglobulin G antibody response to the Sputnik V vaccine: previous SARS-CoV-2 seropositive individuals may need just one vaccine dose. Int J Infect Dis, 111, 261-266. https://doi.org/10.1016/j.ijid.2021.07.070

Else, H. (2020). How a torrent of COVID science changed research publishing - in seven charts. Nature, 588, 553.

Goldberger, A. L., Amaral, L. A., Hausdorff, J. M., Ivanov, P., Peng, C. K., \& Stanley, H. E. (2002). Fractal dynamics in physiology: alterations with disease and aging. Proc Natl Acad Sci U S A, 99 Suppl 1, 2466-2472. https://doi.org/10.1073/pnas.012579499

Hacisuleyman, E., Hale, C., Saito, Y., Blachere, N. E., Bergh, M., Conlon, E. G., Schaefer-Babajew, D. J., DaSilva, J., Muecksch, F., Gaebler, C., Lifton, R., Nussenzweig, M. C., Hatziioannou, T., Bieniasz, P. D., \& Darnell, R. B. (2021). Vaccine Breakthrough Infections with SARS-CoV-2 Variants. N Engl J Med, 384(23), 2212-2218. https://doi.org/10.1056/NEJMoa2105000

Hirsch, J. E. (2005). An index to quantify an individual's scientific research output. Proceedings of the National Academy of Sciences, 102(46), 16569-16572. https://doi.org/10.1073/pnas.0507655102

Jaspe, R. C., Loureiro, C. L., Sulbaran, Y., Moros, Z. C., D'Angelo, P., Rodríguez, L., Zambrano, J. L., Hidalgo, M., Vizzi, E., Alarcón, V., Aguilar, M., Garzaro, D. J., Báez-Ramírez, E., Camacho, A., Delgado, M., Fuentes, Y., Franco, C., Moya, M., Navas-Gil, V. H., . . Pujol, F. H. (2021). Introduction and rapid dissemination of SARSCoV-2 Gamma Variant of Concern in Venezuela. Infection, Genetics and Evolution, 96, 105147. https://doi.org/https://doi.org/10.1016/j.meegid.2021.105147

Liu, Y. C., Kuo, R. L., \& Shih, S. R. (2020). COVID-19: The first documented coronavirus pandemic in history. Biomed J, 43(4), 328-333. https://doi.org/10.1016/j.bj.2020.04.007 
Loureiro, C. L., Jaspe, R. C., P, D. A., Zambrano, J. L., Rodriguez, L., Alarcon, V., Delgado, M., Aguilar, M., Garzaro, D., Rangel, H. R., \& Pujol, F. H. (2021). SARS-CoV-2 genetic diversity in Venezuela: Predominance of D614G variants and analysis of one outbreak. PloS One, 16(2), e0247196.

https://doi.org/10.1371/journal.pone.0247196

Loyo, E. S. L., González, M. J., \& Esparza, J. (2021). Venezuela is collapsing without COVID-19 vaccines. The Lancet, 397(10287), 1806. https://doi.org/10.1016/S0140-6736(21)00924-7

Mehta, N., \& Shukla, S. (2022). Pandemic Analytics: How Countries are Leveraging Big Data Analytics and Artificial Intelligence to Fight COVID-19? SN Comput Sci, 3(1), 54. https://doi.org/10.1007/s42979-02100923-y

Ortega, J. T., Pujol, F. H., Jastrzebska, B., \& Rangel, H. R. (2021). Mutations in the SARS-CoV-2 spike protein modulate the virus affinity to the human ACE2 receptor, an in silico analysis. Excli j, 20, 585-600. https://doi.org/10.17179/excli2021-3471

Petersen, E., Ntoumi, F., Hui, D. S., Abubakar, A., Kramer, L. D., Obiero, C., Tambyah, P. A., Blumberg, L., Yapi, R., Al-Abri, S., Pinto, T. C. A., Yeboah-Manu, D., Haider, N., Asogun, D., Velavan, T. P., Kapata, N., Bates, M., Ansumana, R., Montaldo, C., .. . Zumla, A. (2021). Emergence of new SARS-CoV-2 Variant of Concern Omicron (B.1.1.529) - highlights Africa's research capabilities, but exposes major knowledge gaps, inequities of vaccine distribution, inadequacies in global COVID-19 response and control efforts. Int J Infect Dis, 114, 268-272. https://doi.org/10.1016/j.ijid.2021.11.040

Rangel-Aldao, R., Cruz, L, Jlmenez, I. (2021). El Portal Digital de la Academia Nacional de Medicina de Venezuela: Memoria Descriptiva desde su Gestación (2018-2019), hasta su Puesta en Marcha y Evolución (2020-2021). In E. S. Lopez-Loyo, Urbina-Medina, H. (Ed.), Colección Razetti (Vol. XXV, pp. 257-295). Academia Nacional de Medicina/Editorial ATEPROCA.

Rosillón, D. (2021). Vacunas COVID-19 y lanctancia materna. Gac Méd Car., 129, S648-S651.

Sanchez-Traslaviña, L., et al. (2021). Pacientes hospitalizados con COVID-19: Recuento de un año de pandemia. Gac Méd Car., 129(3), 613-624.

https://drive.google.com/file/d/1kZQECk_UqVIUV_d5wC9qf7nMnZyiBrNG/view

Taleghani, N., \& Taghipour, F. (2021). Diagnosis of COVID-19 for controlling the pandemic: A review of the state-of-the-art. Biosens Bioelectron, 174, 112830. https://doi.org/10.1016/j.bios.2020.112830

Tapia, F. J., Rodríguez, O.L. (2020). Descifrando la patogenia de la COVID-19. Gac Méd Car., 128(3), 307-312. https://drive.google.com/file/d/1qhu3Epubm47ogbvy3TRtGP98hv94upR3/view

Topol, E. J. (2019). High-performance medicine: the convergence of human and artificial intelligence. Nature Medicine, 25(1), 44-56. https://doi.org/10.1038/s41591-018-0300-7

Woldemeskel, B. A., Garliss, C. C., \& Blankson, J. N. (2021). SARS-CoV-2 mRNA vaccines induce broad CD4+ T cell responses that recognize SARS-CoV-2 variants and HCoV-NL63. Journal of Clinical Investigation. https://doi.org/10.1172/jci149335

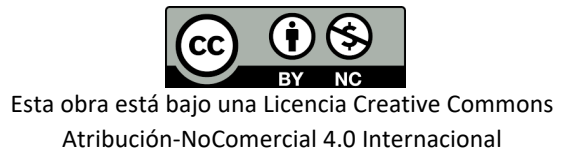

